\title{
The Cost-Effectiveness of Using Epoetin-Beta Versus Darbepoetin-Alfa for the Treatment of Anemia Among Chronic Hemodialysis Patients
}

\author{
Hanan AlKharboush ${ }^{1}$, Fatimah Alshehri ${ }^{1}$, Ibrahim Alatwi ${ }^{2}$, Khaled Al Karni ${ }^{3}$, Abdurahman Alatawi ${ }^{2}$,
} Ahmed M. Hamdan ${ }^{4}$

\begin{abstract}
1. Pharm D Program, Faculty of Pharmacy, University of Tabuk, Tabuk, SAU 2. Pharmaceutical Care Administration, General Directorate of Health Affairs in Tabuk Region, Ministry of Health, Saudi Arabia, Tabuk, SAU 3. Department of Pharmaceutical Care, King Khaled Civil Hospital, Tabuk, SAU 4. Pharmacy Practice, Faculty of Pharmacy, University of Tabuk, Tabuk, SAU
\end{abstract}

Corresponding author: Ahmed M. Hamdan, a_hamdan@ut.edu.sa

\section{Abstract \\ Background and objective}

Anemia is a common prognosis of chronic kidney disease (CKD). It is predominantly managed with synthetic erythropoietin. The principal objective of this study was to compare the cost-effectiveness of the use of short-acting erythropoietin with the long-acting one to maintain serum hemoglobin $(\mathrm{Hb})$ concentration within the range of $10.5-12 \mathrm{~g} / \mathrm{dL}$.

\section{Method}

This was a retrospective cohort study involving patients diagnosed with stage 5 CKD according to the Saudi Society of Nephrology and Transplantation conducted at eight tertiary care centers in the Tabuk region, Saudi Arabia. We compared the cost-effectiveness of long-acting erythropoietin with the short-acting one. The decision analysis model and Markov model were established to simulate a cohort of 55-year-old patients to estimate the incremental cost and quality-adjusted life-year (QALY) for chronic hemodialysis patients (CHP) treated with either darbepoetin-alfa or epoetin-beta for at least nine months. The incremental cost per QALY was the main outcome marker for using both medications. Serum HB levels were monitored on a monthly basis and costs were calculated.

\section{Results}

A total of 291 CHP met our inclusion criteria; 194 of them were treated with darbepoetin-alfa while 97 were treated with epoetin-beta. The mean age was $56.3 \pm 11.2$ years for the darbepoetin-alfa group and $55.2 \pm 7.8$ years for the epoetin-beta cohort. The baseline serum $\mathrm{Hb}$ was $10.68 \pm 0.98 \mathrm{~g} / \mathrm{dL}$ for darbepoetin-alfa patients and $11.63 \pm 0.32 \mathrm{~g} / \mathrm{dL}$ for the epoetin-beta group $(\mathrm{p}=0.003)$. We observed a significant difference between the percentage of patients successfully treated with epoetin-beta and those managed with darbepoetinalfa ( $80.4 \%$ vs. $63.92 \%, p=0.01$ ) with considerably less cardiovascular side effects. The average annual cost per patient was estimated at $\$ 919.47$ and $\$ 12,319.41$ for epoetin-beta and darbepoetin-alfa respectively. Also, the average effectiveness was 0.58 for darbepoetin-alfa vs. 0.61 for epoetin-beta. The average costeffectiveness ratio was $\$ 980.25$ and $\$ 15,023.66$ with an incremental cost difference of $-\$ 966$ in favor of epoetin-beta compared to darbepoetin-alfa.

AlKharboush et al. This is an open access article distributed under the terms of the Creative Commons Attribution License CC-BY 4.0., which permits unrestricted use, distribution, and reproduction in any medium, provided the original author and source are credited.

\section{Conclusion}

Based on our findings, treating anemia in hemodialysis patients using epoetin-beta is very cost-effective compared to managing them with darbepoetin-alfa.

Categories: Nephrology, Epidemiology/Public Health

Keywords: darbepoetin-alfa, epoetin-beta, chronic kidney disease, anemia, cost effective analysis

\section{Introduction}

Chronic kidney disease (CKD) has become a focal point in the debate about improving public health all over the world. Globally, 1.4 million patients receive renal replacement treatment with more than one million patients dying yearly from end-stage renal disease (ESRD) [1]. Lately, ESRD has been found associated with a several-fold increase in mortality and morbidity, especially pertaining to the risk of cardiovascular diseases. Today, ESRD is regarded as a public health issue, and it imposes great human suffering and puts a catastrophic economic burden, mainly on poor countries, where less than $2 \%$ of the ESRD patients have access to renal replacement therapy. Thus, this leads to premature deaths, particularly among those diagnosed with ESRD at the age of less than 20 years [2]. Moreover, an increase in ESRD rates in the future has been predicted, where it will be more prominent in low-income countries, 
Healthcare system planning requires careful assessment of patients with chronic diseases. The epidemiology and treatment of CKD need a careful study regarding its morbidity and mortality rates. The incidence of this disease is on the rise globally [1]. It has been estimated that the global prevalence of CKD is between 11-13\% [2]. These patients suffer from renal anemia, especially in the end stages of the disease, since their kidneys cannot secrete enough erythropoietin to produce red blood cells (RBCs) [3]. Protocols of treatment include RBC transfusion (RBCT) or erythropoietin-stimulating agents (ESA) such as epoetin-alfa, epoetin-beta, and darbepoetin-alfa [4]. ESAs are a group of medications that are directed towards bone marrow in order to stimulate the differentiation of progenitor cells to RBCs in the treatment of anemia associated with endstage CKD. Every ESA requires continuous administration to keep stable serum hemoglobin (Hb) levels in the desired range. In 2017, it was reported that there are between 2,201,128 to 2,595,630 patients in Saudi Arabia with a mortality rate of nearly $9.9 \%[1]$.

The risk factors for the incidence of CKD in Saudi Arabia are highly diverse and the nature of the risk is very high. They include hypertension, diabetes, and obesity [5], which are prevalent in Saudi Arabia, especially in the northwestern area: the Tabuk region. The Tabuk region consists of five governorates including approximately 170 villages. According to the official records, the population in Tabuk is estimated to be around one million [6]. There are eight hemodialysis centers with varying capacities in the region; seven are affiliated with the Ministry of Health and one is under private management (DaVita Center). Both RBCT and ESA are used for treating CKD in these centers. It has been reported that using ESA leads to greater improvement in the quality of life (QOL) and quality-adjusted life-year (QALY) compared with RBCT [7-9]. Hence, ESA has become the standard option for treating CKD. ESA class includes short-acting medications such as epoetin-beta and long-acting ones such as darbepoetin-alfa. The principal objective of this study is to compare the cost-effectiveness of these two medications (epoetin beta and darbepoetin-alfa) for managing anemia associated with CKD. Previous health economic studies mainly focused on short-acting medications [10-14]. Moreover, the health outcomes comparing short- and long-acting ESAs and their effects on cost have not been extensively investigated yet in the Tabuk region. In light of this, we performed a retrospective cohort study in order to do a cost-effectiveness analysis from the payer perspective to estimate the potential impact of using two treatment strategies for anemia associated with CKD: shortacting erythropoietin: epoetin-beta three times weekly vs. long-acting erythropoietin: darbepoetin-alfa once monthly. We believe this study can serve as a guide for decision-makers, especially those at the Saudi Society of Nephrology and Transplantation, for devising treatment protocols for chronic hemodialysis patients (CHP).

\section{Materials And Methods}

\section{Study design}

We selected patient files of patients admitted to the hemodialysis units and diagnosed with CDK stage 5 with anemia from the hospitals' clinical data registry entry from eight tertiary care hospitals in the Tabuk region: Tabuk city (King Khaled Civil Hospital, DaVita Dialysis Center), Haqel, Diba, Alwajh, Albada, Tayma, and Umluj; these admissions spanned a period of one year from January to December 2019. The collected data included the prescribed medications with their doses along with therapeutic outcomes in terms of the monthly follow-up change in serum $\mathrm{Hb}$ levels to reach the therapeutic targeted level (10.5-12 g/dL) and the reported side effects that emerged during the course of treatment (cardiovascular side effects). Afterward, we applied the decision analysis model and Markov model to simulate a cohort of 55-year-old patients to estimate the incremental cost and QALY for CHP treated with either darbepoetin-alfa or epoetin-beta for at least nine months.

\section{The inclusion criteria}

The inclusion criteria were as follows: all CHP diagnosed with stage 5 CKD (both Saudi and non-Saudi residents in the Tabuk region only; not transferred from other kidney care units outside the Tabuk region) who were above 18 years old with $\mathrm{CKD}$ on hemodialysis therapy for at least nine months with the same mode of dialysis and same dosing interval for at least three months. Patients in all centers except DaVita had to be on darbepoetin-alfa (50 or $100 \mathrm{mcg} / 0.5 \mathrm{~mL}$ ) once monthly. DaVita Center was using epoetin-beta 1,000 IU thrice weekly. These choices had already been decided by the policies of the healthcare payers, who were the providers of ESA for all patients, and in accordance with physician directions.

We used the laboratory parameters measured on a monthly basis: Kt/V, serum $\mathrm{Hb}$ concentration, serum transferrin, transferrin saturation (TSAT), RBC count, hematocrit, platelet count, ferritin, serum intact parathyroid hormone (iPTH), albumin, phosphate concentration, and calcium concentration to follow up the patients. We used a serum $\mathrm{Hb}$ level between 10.5-12 g/dL as the target for our cost-effectiveness analysis.

\section{The exclusion criteria}

We excluded patients who had received organ transplantation, those who did not complete at least nine months of continuous same therapy, and those who had become anemic due to reasons other than CKD. 


\section{Ethical considerations}

All procedures were performed in accordance with the ethical standards of the institutional and national research ethics committee (no: UT-99-03-2020) from the University of Tabuk and (TU-077/019/015) 529871441 from the Research and Study Center at the Saudi Ministry of Health.

\section{The cost-effective analysis}

We used the data for analysis according to the healthcare payer perspective. Moreover, we used the decisionanalytic technique to model this situation and Markov's model for evaluation of the average incremental cost of the two medications to treat anemia. The Markov model consisted of four health conditions for each patient, as shown in Figure 1, according to the serum Hb levels; we then compared each condition with a serum $\mathrm{Hb}$ level of less than $9 \mathrm{~g} / \mathrm{dL}$.

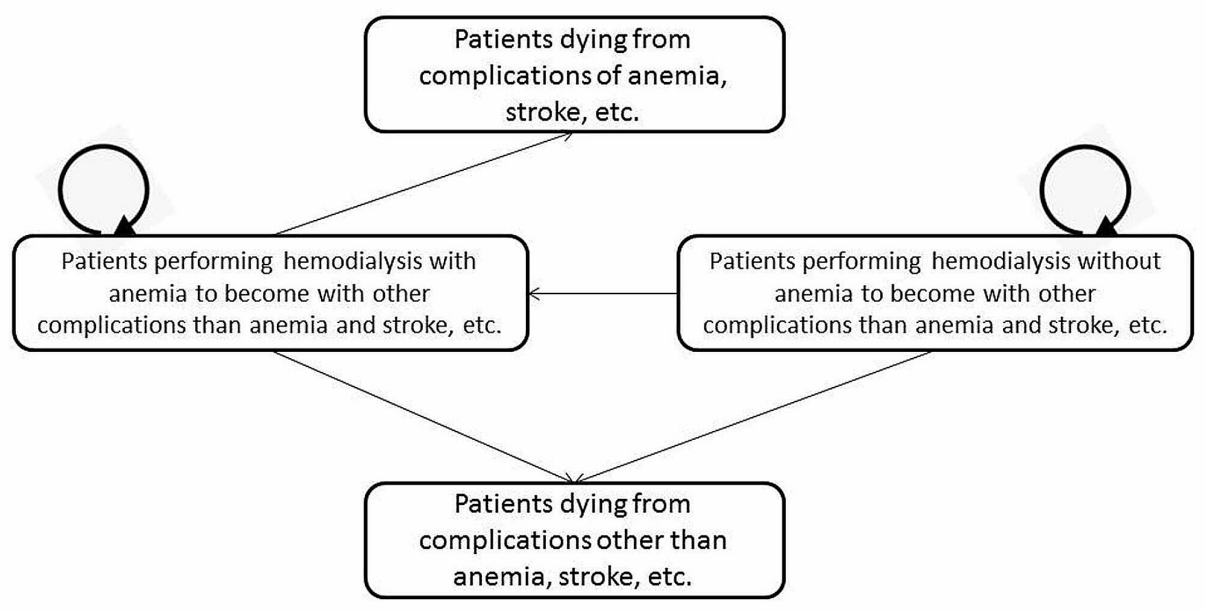

FIGURE 1: Schematic presentation of the Markov model for the treatment of ESRD patients with erythropoietin

ESRD: end-stage renal disease

Firstly, for patients who were alive and undergoing hemodialysis and suffering from anemia, stroke, etc., we assigned a serum $\mathrm{Hb}$ level of 9-10 g/dL. Secondly, for patients who were alive and undergoing hemodialysis and suffering from any other problem such as catheter infection, we assigned a serum Hb level of 10-12 g/dL. Thirdly, for patients who were dying and suffering from complications of anemia, stroke, etc., we assigned a serum $\mathrm{Hb}$ level of more than $13 \mathrm{~g} / \mathrm{dL}$. Finally, for patients who were dying and suffering from other complications, we assigned a serum Hb level of 12-13 g/dL. This Markov model had a cycle with a length of four weeks and a lifetime horizon. We used the four-weekly measured targeted serum $\mathrm{Hb}$ concentration between 10.5-12 g/dL as the target for both treatments. Those with Hb levels higher than 12 $\mathrm{g} / \mathrm{dL}$ or lower than $10.5 \mathrm{~g} / \mathrm{dL}$ were considered as one group and called "patients out of targeted serum $\mathrm{Hb}$." Clinical success rate (CSR) is the proportion of the population achieving the targeted serum $\mathrm{Hb}$ concentration. We assumed that the patients would continue to perform hemodialysis until their death. The movement between each health state was assumed to be dependent on the serum $\mathrm{Hb}$ level. They were the probabilities that determined the transition between each health status. Costs and QALYs gained were calculated as patients went through this model.

\section{QALY calculation}

Calculation of QALYs was performed using the additional number of years of life gained from an intervention multiplied by a utility judgment of the QOL. The QALYs gained can be calculated using the probabilities to determine the mean, variance, and probability distribution for the QALYs gained. The Markov model was established to simulate a cohort of 55-year-old people. It was carried out using TreeAge Pro 2020 (TreeAge Software, Inc., Williamstown, MA).

The cost elements in Saudi Riyals in reports for every month were converted to US Dollars (\$) (one US Dollar equals 3.77 Saudi Riyal). We applied the Markov model of a hypothetical cohort of dialysis patients treated with epoetin-beta three times weekly or darbepoetin-alfa once monthly for one year. We applied the decision-analytical technique using TreeAge Pro 2020.

\section{Assumptions}


For model inputs, we assumed that there was no change related to hospitalizations between the two treatments (no extra professional visits, advice, oxidative stress, etc.), no difference between surveillance costs between the two treatments, and no difference between the two groups of patients regarding hospital visits for hemodialysis irrespective of the treatment type. Moreover, we assumed that the laboratory assays were the same in all hemodialysis centers. Also, we assumed that there was no difference between the serum $\mathrm{Hb}$ target levels between male and female patients. We also assumed that there was no significant dose difference between the two medications given to patients with central nervous catheters and those taking them using arteriovenous fistula.

\section{Sensitivity analysis and Monte Carlo simulation}

Our model included assumptions and uncertainty. Hence, we performed a one-way sensitivity analysis for the individual variation and multi-way sensitivity analysis variation of multi-components at the same time, by varying baseline estimation among the ranges of potentially reasonable values such as a number of patients in the hospitals, the length of stay in hospitals, survival estimates, ESA costs, utilities, etc. Moreover, a probabilistic sensitivity analysis was conducted by a Monte Carlo simulation (MCS) to monitor and to eliminate the uncertainty by creating 50,000 samples. Afterward, we calculated the expected value. Using that model, the expected value was calculated to test and to evaluate whether these changes modified the reached conclusion. Normal distribution of clinical parameters was employed for the relative and baseline risk. A log-normal distribution was applied to the cost estimates.

\section{Statistical analysis}

Statistical analyses were done and groups were compared using SPSS Statistics software 24.0 (IBM, Armonk, NY). Depending on the variable distributions between the two groups, we used the Mann-Whitney test or Wilcoxon test. All results of non-normally distributed variables were presented as percentages of discrete variables. A p-value of $<0.05$ was considered statistically significant.

\section{Results}

\section{Patient cohort characteristics}

We initially screened 341 patients in total; 291 CHP met our inclusion criteria and were deemed eligible for analysis (85.34\%). Among them, $194 \mathrm{CHP}$ were treated with darbepoetin-alfa, with a mean age of $56.3 \pm 11.2$ years, and 97 CHP were treated with epoetin-beta, with a mean age of $55.2 \pm 7.8$ years (Table 1). 


\section{Cureus}

\begin{tabular}{|c|c|c|c|}
\hline Variables & Darbepoetin-alfa & Epoetin-beta & P-value* \\
\hline Number of CHP & 194 & 97 & \\
\hline Age in years, mean \pm SD & $56.3 \pm 11.2$ & $55.2 \pm 7.8$ & 0.53 \\
\hline Male, n (\%) & $99(51 \%)$ & $50(51.55 \%)$ & 0.69 \\
\hline Primary renal disease, $\mathrm{n}(\%)$ & $62(32 \%)$ & $19(19.6 \%)$ & 0.01 \\
\hline Diabetes, n (\%) & $44(22.7 \%)$ & 32 (32.9\%) & 0.01 \\
\hline Hypertension, n (\%) & $29(14.9 \%)$ & $19(19.6 \%)$ & 0.02 \\
\hline Obesity, n (\%) & $17(8.8 \%)$ & $17(17.5 \%)$ & 0.02 \\
\hline Other, n (\%) & $42(21.6 \%)$ & $10(10.3 \%)$ & 0.08 \\
\hline Time of dialysis (hours), mean \pm SD & $410 \pm 24.3$ & $391 \pm 9.8$ & 0.034 \\
\hline \multicolumn{4}{|l|}{ Pre-dialysis blood pressure } \\
\hline Systolic BP (mmHg), mean \pm SD & $124 \pm 26.9$ & $131 \pm 12.9$ & 0.52 \\
\hline Diastolic BP $(\mathrm{mmHg})$, mean \pm SD & $79 \pm 1.3$ & $80 \pm 1.9$ & 0.51 \\
\hline \multicolumn{4}{|l|}{ Laboratory results } \\
\hline Hemoglobin (g/dL), mean \pm SD & $10.68 \pm 0.98$ & $11.63 \pm 0.32$ & 0.03 \\
\hline Albumin (g/L), mean \pm SD & $3.9 \pm 0.91$ & $3.9 \pm 0.89$ & 0.51 \\
\hline Ferritin $(\mathrm{ng} / \mathrm{mL})$, mean $\pm \mathrm{SD}$ & $352.91 \pm 53$ & $362.12 \pm 129$ & 0.63 \\
\hline Calcium (mg/dL), mean \pm SD & $9.81 \pm 0.23$ & $9.8 \pm 0.24$ & 0.74 \\
\hline Phosphate $(\mathrm{mg} / \mathrm{dL})$, mean $\pm \mathrm{SD}$ & $4.4 \pm 1.21$ & $4.2 \pm 1.11$ & 0.62 \\
\hline Triglyceride (mg/dL), mean \pm SD & $151.3 \pm 33.2$ & $142 \pm 23.1$ & 0.51 \\
\hline $\mathrm{Kt} / \mathrm{V}$, mean $\pm \mathrm{SD}$ & $1.42 \pm 0.22$ & $1.5 \pm 0.1$ & 0.49 \\
\hline Cardiovascular diseases after taking the medication, $\mathrm{n}(\%)$ & $19(9.79 \%)$ & $3(3.09 \%)$ & 0.005 \\
\hline \multicolumn{4}{|l|}{ Vascular access } \\
\hline Fistula, $n(\%)$ & $182(93.8 \%)$ & $88(90.7 \%)$ & 0.3 \\
\hline Catheter, n (\%) & $12(6.2 \%)$ & $9(9.3 \%)$ & 0.3 \\
\hline
\end{tabular}

TABLE 1: Demographic characteristics and laboratory results of each group of patients treated with short- and long-acting erythropoietin

*Statistical significance at a p-value of $<0.05$

CHP: chronic hemodialysis patients; SD: standard deviation

As shown in Table 1, there was no significant difference between the two groups in terms of age, gender, and laboratory tests other than $\mathrm{Hb}$ level ( $\mathrm{p}=0.03$ for $\mathrm{Hb}$ levels). The numbers meant nothing. Meanwhile, there was a higher burden of accompanying diseases such as diabetes, hypertension, and obesity in the group receiving epoetin-beta $(\mathrm{p}<0.05)(19$ patients on darbepoetin-alfa vs. three patients on epoetin-beta; $\mathrm{p}=0.005$ ). This indicated that patients treated with epoetin-beta were more complicated cases. There was no significant difference between the two groups in the percentage of patients using catheters for hemodialysis. However, there was a significant difference between the two treatment groups in terms of achieving targeted $\mathrm{Hb}$ concentration $(\mathrm{p}=0.03)$.

\section{Efficacy evaluation}

As shown in Table 2, we noticed that the time of dialysis in the epoetin-beta cohort was significantly less than that in the darbepoetin-alfa group: 3.5 hours for darbepoetin-alfa vs. 2.8 hours for epoetin-beta $(\mathrm{p}=0.034)$, with a significant difference between the monthly measured baseline $\mathrm{Hb}$ levels in the two groups 


\section{Cureus}

$(10.68 \pm 0.98 \mathrm{~g} / \mathrm{dL}$ for darbepoetin-alfa vs. $11.63 \pm 0.32 \mathrm{~g} / \mathrm{dL}$ for epoetin-beta, $\mathrm{p}=0.001)$. We noticed significantly reduced cardiovascular problems in patients taking epoetin-beta compared to those taking darbepoetin-alfa $(\mathrm{p}=0.05)$. Moreover, we found that a significantly less number of patients with lower HB levels ( $<10.5 \mathrm{~g} / \mathrm{dL}$ ) or higher Hb levels ( $>12 \mathrm{~g} / \mathrm{dL}$ ) used epoetin-beta $(19.6 \%$ and $36.08 \%$ for epoetin-beta and darbepoetin-alfa respectively). Hence, the relative risk reduction (RRR) of using epoetin-beta compared to using darbepoetin-alfa was $16.48 \%$ for the risk of not obtaining the required $\mathrm{Hb}$ range level. The effectiveness was $80.4 \%$ and $63.92 \%$ for epoetin-beta and darbepoetin-alfa respectively to achieve our target monthly measured $\mathrm{Hb}$ levels. So, the cost-effectiveness was $\$ 980.25$ and $\$ 15,023.66$ for epoetin-beta and darbepoetin-alfa respectively to treat one patient per month successfully. Hence, the incremental cost was about $-\$ 966$ per additional case to successfully achieve the required range of monthly measured $\mathrm{Hb}$ level.

\begin{tabular}{|c|c|c|c|}
\hline Parameter & Darbepoetin-alfa & Epoetin-beta & P-value* \\
\hline Number of patients & 194 & 97 & \\
\hline $\mathrm{Hb}$ level (g/dL), mean $\pm \mathrm{SD}$ & $10.68 \pm 0.98$ & $11.63 \pm 0.32$ & 0.03 \\
\hline Transferrin saturation (\%) & 26.9 & 29.2 & 0.04 \\
\hline Ferritin (ng/mL) & 287.9 & 298.6 & 0.42 \\
\hline IV iron (\%) & 83.9 & 88.9 & 0.59 \\
\hline IV iron dosage (mg/month), mean \pm SD & $110.3 \pm 11.3$ & $115.9 \pm 19.1$ & 0.51 \\
\hline Drug dosage, mean $\pm S D$ & $5,980 \pm 2,987$ IU & $98.4 \pm 39.8 \mu \mathrm{g}$ & \\
\hline CSR, n (\%) & $124(63.92 \%)$ & $78(80.4 \%)$ & 0.02 \\
\hline Patients with $\mathrm{Hb}$ of $>12 \mathrm{~g} / \mathrm{dL}$ or $<10.5 \mathrm{~g} / \mathrm{dL}, \mathrm{n}(\%)$ & $70(36.08 \%)$ & $19(19.6 \%)$ & 0.01 \\
\hline Dialysis time per patient per session (hours) & 3.5 & 2.8 & 0.06 \\
\hline Cardiovascular diseases after taking the medication, $\mathrm{n}$ (\%) & $19(9.79 \%)$ & $3(3.09 \%)$ & 0.05 \\
\hline
\end{tabular}

\section{TABLE 2: Laboratory analysis of patients treated with either epoetin-beta or darbepoetin-alfa}

*Statistical significance at a p-value of $<0.05$

Hb: hemoglobin; IV: intravenous; CSR: clinical success rate; SD: standard deviation

\section{Baseline clinical costs and input assumptions}

As shown in Table 3, over a time horizon of one year, we considered the direct medical costs for both epoetin-beta and darbepoetin-alfa including fees for medical consultant, medical specialist, nutritional specialist, pharmacist, and social specialist, and the cost incurred for iron usage, medication usage, and hospitalization. We assumed that there was no significant difference in terms of hospitalization and the length of stay for both medications. The average annual cost per patient was estimated at $\$ 919.47$ and $\$ 12,319.41$ for epoetin-beta and darbepoetin-alfa respectively. There was no statistically significant difference between using the two medications in the monthly monitored parameters, as shown in Table 1 . The cost-saving associated with using epoetin-beta was $\$ 11,399.95$. The average annual effectiveness estimated using our model was 0.58 and 0.61 for darbepoetin-alfa and epoetin-beta respectively. So, the average cost-effectiveness was $\$ 533.29$ and $\$ 713,886.05$ for epoetin-beta and darbepoetin-alfa respectively (Figure 2). 


\section{Cureus}

\section{Variables}

Medical consultant

Medical specialist

Nutritional specialist

Pharmacist

Social specialist

Darbepoetin-alfa cost

Epoetin-beta cost

Hospitalization for darbepoetin-alfa

Hospitalization for epoetin-beta
Base-case estimate per patient per year (\$)

$3,405.6$

$2,311.2$

1,542

$1,455.6$

$1,180.8$

$6,638.1$

915.2

$19,050.759$

$19,155.7984$

TABLE 3: Baseline clinical costs per month and input assumptions

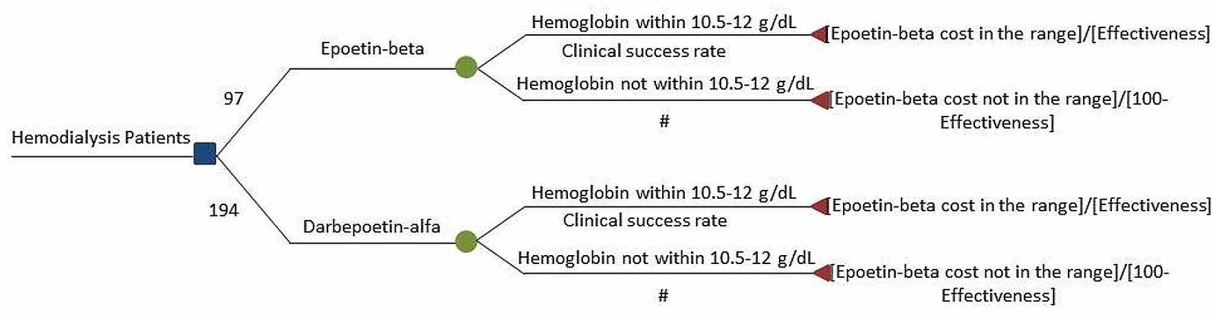

(a)

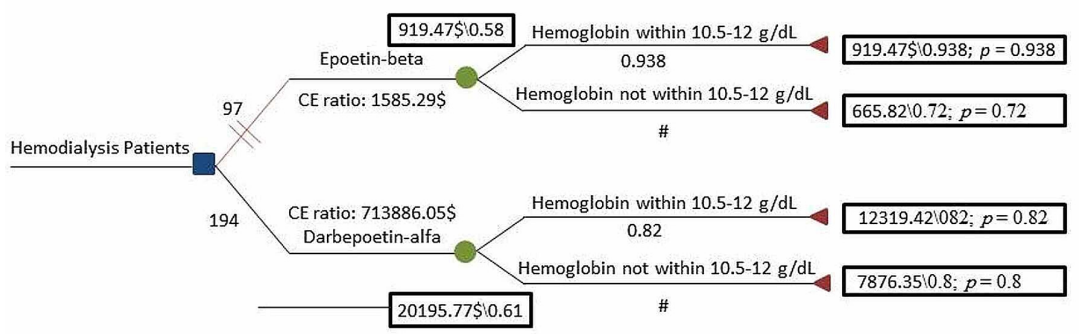

(b)

FIGURE 2: Cost-effectiveness analysis of epoetin-beta vs darbepoetinalfa in the treatment of anemia associated with CKD in hemodialysis patients

(a) Decision tree framework. (b) Results of cost-effectiveness analysis after roll-back calculation

CKD: chronic kidney disease

Additionally, the treatment of anemia associated with hemodialysis CKD patients with epoetin-beta dominated darbepoetin-alfa as shown in the cost-effectiveness diagram (Figure 3). 


\section{Cureus}

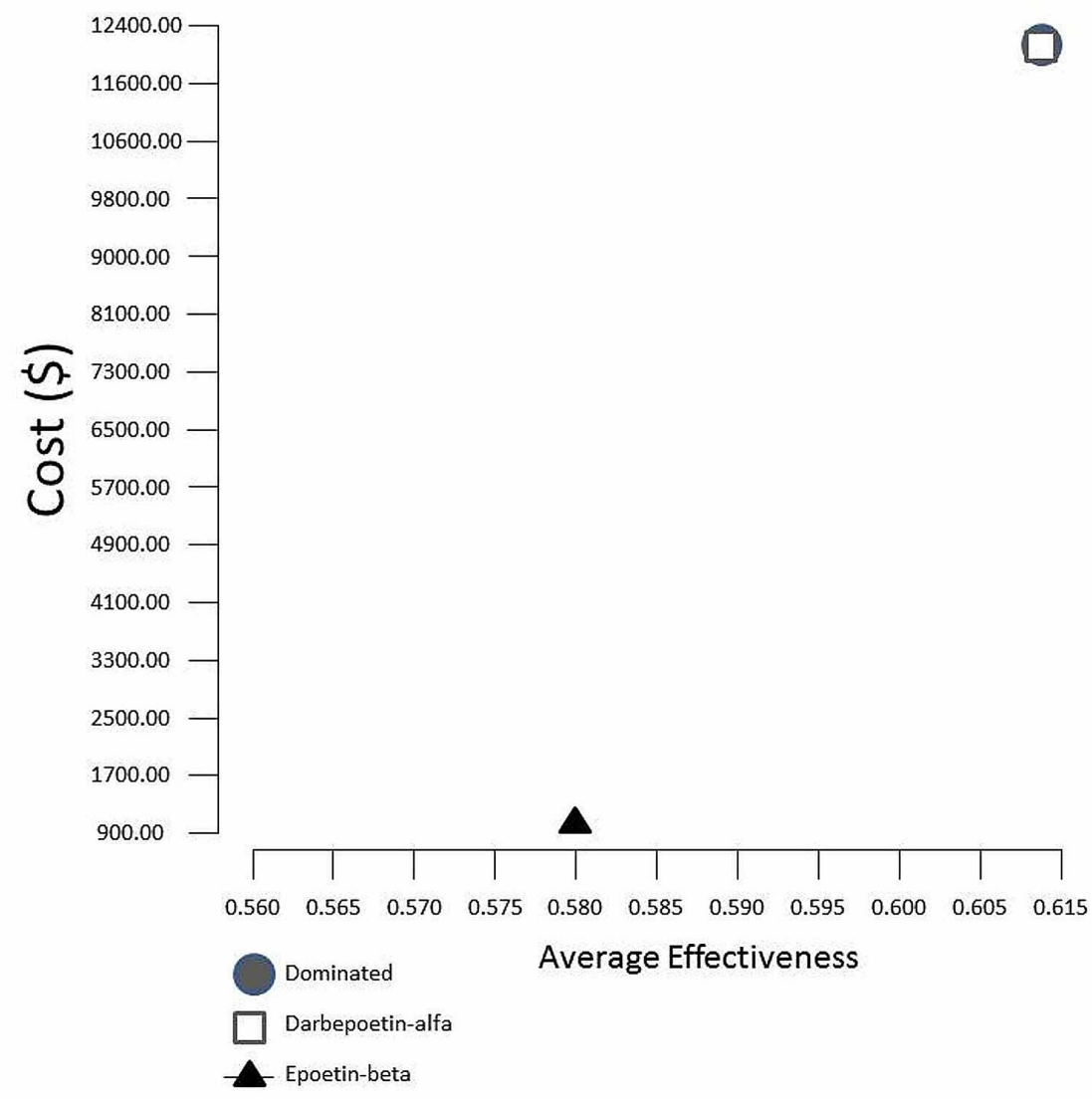

\section{FIGURE 3: Cost-effectiveness diagram}

Epoetin-beta dominated darbepoetin-alfa since it is highly effective and less costly

\section{Quality-adjusted life-year}

QALY is a tool used for evaluating the health benefits of using medications. So, we used QALY analysis to examine preferential therapeutic outcomes of using one medication over the other. Moreover, we calculated the incremental cost per additional QALY. In Table 4, cost, QALYs, and incremental cost over a one-year time horizon associated with epoetin-beta dominating darbepoetin-alfa administration are summarized. The average annual cost per patient was estimated at $\$ 919.47$ and $\$ 12,319.41$ for epoetin-beta and darbepoetinalfa respectively. In our model, epoetin-beta administration was associated with an increase in patients' QOL by 0.1 QALYs per additional life-years gained on average compared with darbepoetin-alfa over the lifetime horizon with an incremental cost of $\$ 12,297.86$ for darbepoetin-alfa and $\$ 897.92$ for epoetin-beta. Therefore, the cost-utility ratio was calculated at 20,845.01 and 1,519.32 \$/OALY for darbepoetin-alfa and epoetin-beta respectively, with an incremental cost-utility ratio (ICUR) of darbepoetin-alfa and epoetinbeta at 6,1597.05 and 4,597.35 \$/QALY respectively.

\begin{tabular}{|c|c|c|c|c|c|c|}
\hline Intervention & Average cost (\$) & Incremental cost (\$) & Average QALY & Incremental cost & Cost-utility ratio & ICUR (\$/QALY) \\
\hline Darbepoetin-alfa & $86,135.1$ & $12,297.86$ & 0.591 & 1,273 & $20,845.01$ & $6,1597.05$ \\
\hline Epoetin-beta & $20,774.3$ & 897.92 & 0.591 & 869 & $1,519.32$ & $4,597.35$ \\
\hline
\end{tabular}

TABLE 4: Costs, QALY, and incremental cost per year

QALY: quality-adjusted life-year; ICUR: incremental cost-utility ratio

For the one-way sensitivity analysis, the inputs in the model were allowed to vary within clinically plausible ranges. Table 4 shows the sensitivity of the base-case ICUR of darbepoetin-alfa and epoetin-beta in different scenarios tested. The model was most sensitive to hospitalization costs and hospital stay, and any increase in those parameters resulted in a decrease in ICURs as reported in Table 4. Assuming utility and survival 
improvement with darbepoetin-alfa and epoetin-beta use while all costs remained the same resulted in a decrease in ICUR at 61,597.05 \$/QALY for darbepoetin-alfa and 4,597.35 \$/QALY for epoetin-beta. Testing the hypothesis of equal mortality rate between darbepoetin-alfa and epoetin-beta use resulted in an ICUR at 20,878 \$/QALY and 23,940 \$/QALY respectively. Decreasing the acquisition cost of darbepoetin-alfa and epoetin-beta by $25 \%$ resulted in an ICUR at 11,911 \$/QALY and 14,088 \$/QALY respectively.

\section{Sensitivity analysis and Monte Carlo simulation}

We did a one-way and multiway sensitivity study of the results by MCS. For this analysis, 50,000 random simulated trials were run (Figure 4).

\section{Incremental Effectiveness}

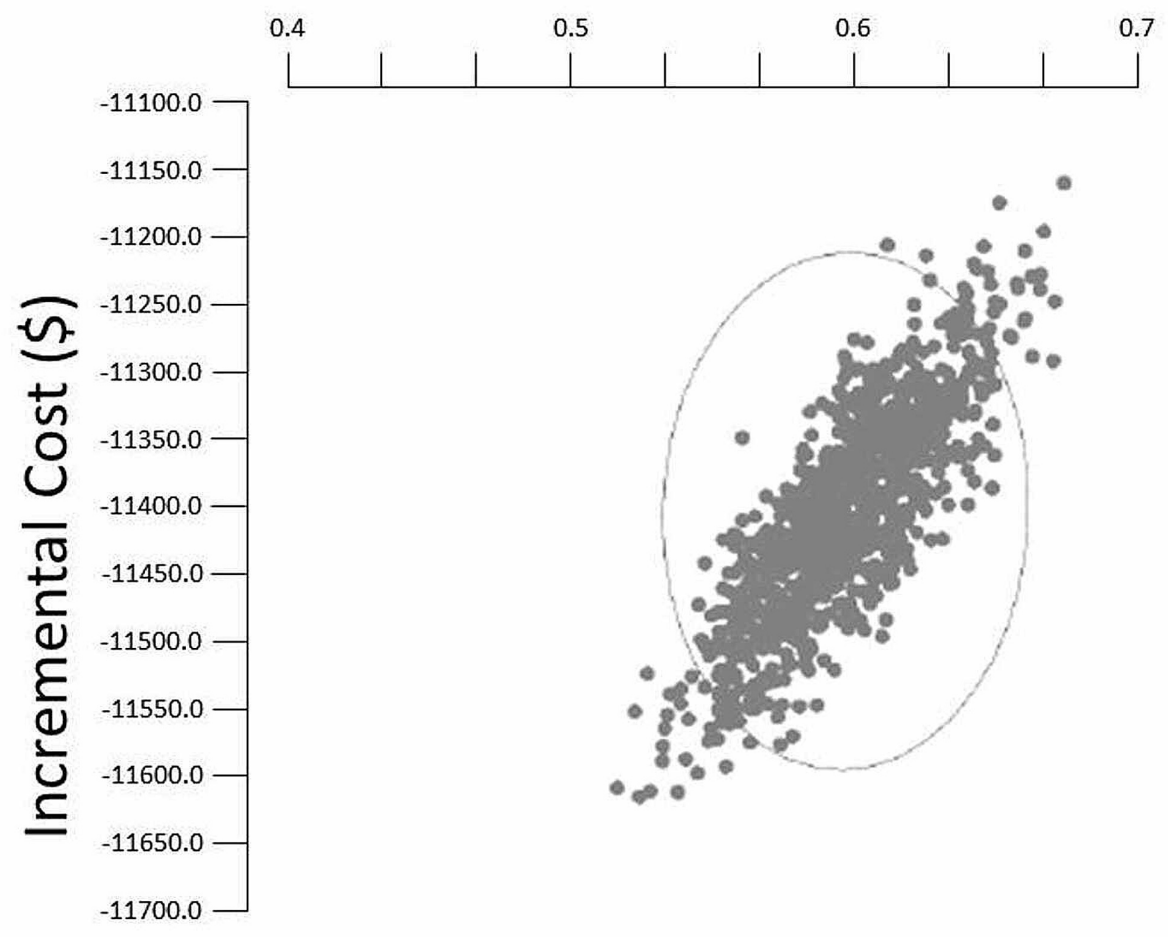

FIGURE 4: Results of Monte Carlo simulation of incremental cost and effectiveness of scattered plot of epoetin-beta versus darbepoetin-alfa based on 50,000 random iterations of the cost-effectiveness model

The scattered plot showed the robustness of the results; $100 \%$ of the results were in the lower right-hand part of the graph (best clinical effect and lower cost). So, epoetin-beta dominated darbepoetin-alfa in the clinical effectiveness parameter with lower cost. Also, the robustness of the calculated ICUR was confirmed by the probabilistic sensitivity analysis distribution of epoetin-beta versus darbepoetin-alfa based on 50,000 random iterations (Figure 5). The analysis showed that the incremental cost between these two medications remained in the range of $10 \%$. 


\section{Cureus}

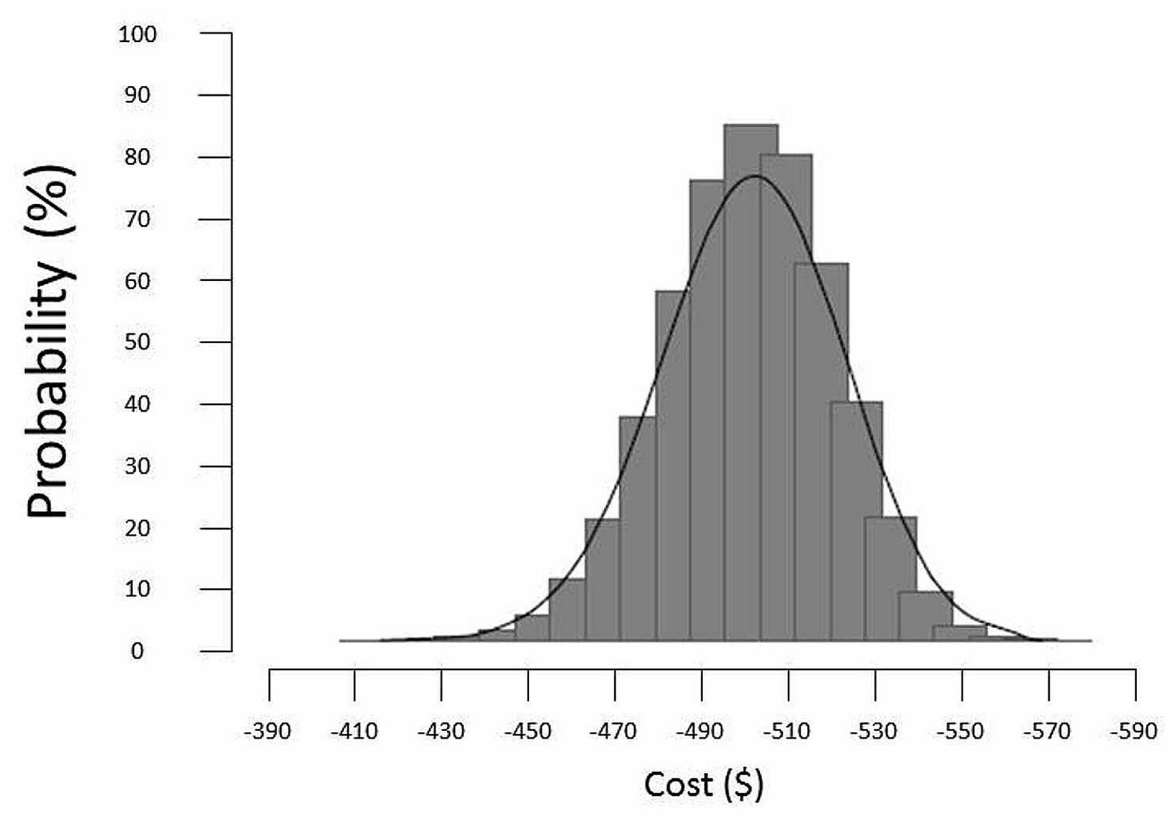

FIGURE 5: Probabilistic sensitivity analysis distribution of epoetin-beta versus darbepoetin-alfa

\section{Discussion}

This research involved a comparison of the potential therapeutic and economic impact of using two erythropoietin drugs (epoetin-beta and darbepoetin-alfa) among patients with stage 5 CKD for the treatment of associated anemia. Our pharmacoeconomic analysis showed that anemia in CKD patients treated with epoetin-beta is associated with a substantial clinical benefit and results in significant additional cost savings relative to darbepoetin-alfa. This finding is consistent with that of most studies [10-16]. Elaborated sensitivity analyses revealed that the healthcare payer should be willing to pay at least $\$ 11,399.95$ (the difference between $\$ 12,297.86$ for darbepoetin-alfa and $\$ 897.92$ for epoetin-beta) per additional QALY for darbepoetin-alfa to become cost-effective compared to epoetin-beta. Moreover, the present study found that epoetin-beta is more cost-effective than darbepoetin-alfa, making it the dominant treatment modality for the management of anemia in CHP.

Our data contrast with the findings of Maoujoud et al. [11], who concluded that darbepoetin-alfa is more effective than epoetin-beta in a prospective cohort study of Moroccan patients. These differences in findings may be due to the genetic variation between our patients and the Moroccan cohort. Moreover, the study involved administering epoetin-beta for six months. After that, they shifted patients to darbepoetinalfa. So, the erythropoietin cycle may need to be longer to get good results. Meanwhile, our data align with that of Schmid [13]. His literature review of studies that dealt with the cost or the cost-effectiveness of darbepoetin-alfa was poor. The main limitation of that study was that he referred to 18 publications in his analysis, and most of the available data was from meeting abstracts (11), and only seven published studies were from peer-reviewed journals. The majority of included studies were retrospectives, and reported data were only about the cost of therapy after a switch to darbepoetin-alfa at a single center. Our study proved that epoetin-beta is superior to darbepoetin-alfa in treating anemia with less cardiovascular side effects based on the data from eight centers of hemodialysis.

\section{Conclusions}

We performed a cost-effectiveness analysis regarding the use of darbepoetin-alfa (long-acting ESA) versus epoetin-beta (short-acting ESA) for the treatment of anemia associated with stage 5 CKD to evaluate their potential outcomes. The findings from our economic model revealed that the use of epoetin-beta was associated with additional cost benefits compared to darbepoetin-alfa. Besides, healthcare payers should be willing to pay at least $\$ 11,399.95$ per additional QALY for darbepoetin-alfa to become cost-effective compared to epoetin-beta. These findings were robust to the sensitivity analysis. Our study also found that the use of epoetin-beta significantly improves the patients' QOL.

\section{Additional Information}

\section{Disclosures}


Human subjects: Consent was obtained by all participants in this study. The Research and Study Center at the Saudi Ministry of Health issued approval TU-077/019/015. This study was approved by the Research and Study Center at the Saudi Ministry of Health. Animal subjects: All authors have confirmed that this study did not involve animal subjects or tissue. Conflicts of interest: In compliance with the ICMJE uniform disclosure form, all authors declare the following: Payment/services info: All authors have declared that no financial support was received from any organization for the submitted work. Financial relationships: All authors have declared that they have no financial relationships at present or within the previous three years with any organizations that might have an interest in the submitted work. Other relationships: All authors have declared that there are no other relationships or activities that could appear to have influenced the submitted work.

\section{References}

1. Alkhlaif AA, Alsuraimi AK, Bawazir AA: Epidemiological profile of end-stage renal diseases in Riyadh, Saudi Arabia. Asian J Med Health. 2020, 18:16-27. 10.9734/AJMAH/2020/v18i730220

2. GBD Chronic Kidney Disease Collaboration: Global, regional, and national burden of chronic kidney disease, 1990-2017: a systematic analysis for the Global Burden of Disease Study 2017. Lancet. 2020, 395:709-733. 10.1016/S0140-6736(20)30045-3

3. Hill NR, Fatoba ST, Oke JL, Hirst JA, O'Callaghan CA, Lasserson DS, Hobbs FD: Global prevalence of chronic kidney disease - a systematic review and meta-analysis. PLoS One. 2016, 11:e0158765. 10.1371/journal.pone. 0158765

4. Cases A, Egocheaga MI, Tranche S, Pallarés V, Ojeda R, Górriz JL, Portolés JM: Anemia of chronic kidney disease: protocol of study, management and referral to nephrology. Nefrologia. 2018, 38:8-12. 10.1016/j.nefro.2017.09.004

5. Mikhail A, Brown C, Williams JA, et al.: Renal association clinical practice guideline on anaemia of chronic kidney disease. BMC Nephrol. 2017, 18:345. 10.1186/s12882-017-0688-1

6. Kazancioğlu R: Risk factors for chronic kidney disease: an update . Kidney Int Suppl (2011). 2013, 3:368-371. 10.1038/kisup.2013.79

7. El Minshawy O, Ghabrah T, El Bassuoni E: End-stage renal disease in Tabuk Area, Saudi Arabia: an epidemiological study. Saudi J Kidney Dis Transpl. 2014, 25:192-195. 10.4103/1319-2442.124574

8. Clement FM, Klarenbach S, Tonelli M, Johnson JA, Manns BJ: The impact of selecting a high hemoglobin target level on health-related quality of life for patients with chronic kidney disease: a systematic review and meta-analysis. Arch Intern Med. 2009, 169:1104-1112. 10.1001/archinternmed.2009.112

9. Remák E, Hutton J, Jones M, Zagari M: Changes in cost-effectiveness over time. The case of epoetin alfa for renal replacement therapy patients in the UK. Eur J Health Econ. 2003, 4:115-121. 10.1007/s10198-0020164-Z

10. Borg S, Glenngård AH, Osterborg A, Persson U: The cost-effectiveness of treatment with erythropoietin compared to red blood cell transfusions for patients with chemotherapy induced anaemia: a Markov model. Acta Oncol. 2008, 47:1009-1017. 10.1080/02841860701744498

11. Maoujoud O, Ahid S, Cherrah Y: The cost-utility of treating anemia with continuous erythropoietin receptor activator or Epoetin versus routine blood transfusions among chronic hemodialysis patients. Int J Nephrol Renovasc Dis. 2016, 9:35-43. 10.2147/IJNRD.S96027

12. Maoujoud O, Ahid S, Dkhissi H, Oualim Z, Cherrah Y: The cost-effectiveness of continuous erythropoiesis receptor activator once monthly versus epoetin thrice weekly for anaemia management in chronic haemodialysis patients. Anemia. 2015, 2015:189404. 10.1155/2015/189404

13. Schmid H: Cost-effectiveness of continuous erythropoietin receptor activator in anemia . Clinicoecon Outcomes Res. 2014, 6:319-330. 10.2147/CEOR.S46930

14. Becker R, Dembek C, White LA, Garrison LP: The cost offsets and cost-effectiveness associated with pegylated drugs: a review of the literature. Expert Rev Pharmacoecon Outcomes Res. 2012, 12:775-793. 10.1586/erp. 12.65

15. Clement FM, Klarenbach S, Tonelli M, Wiebe N, Hemmelgarn B, Manns BJ: An economic evaluation of erythropoiesis-stimulating agents in CKD. Am J Kidney Dis. 2010, 56:1050-1061. 10.1053/j.ajkd.2010.07.015

16. Oh J, Joo KW, Chin HJ, et al.: Correction of anemia with continuous erythropoietin receptor activator in Korean patients on long-term hemodialysis. J Korean Med Sci. 2014, 29:76-83. 10.3346/jkms.2014.29.1.76 